\title{
Highly Distributed XQuery with DXQ
}

\author{
Mary Fernandez Trevor Jim \\ AT\&T Labs Research \\ \{mff,trevor\}@research.att.com \\ Kristi Morton \\ University of Texas Austin \\ kmorton@cs.utexas.edu
}

\author{
Nicola Onose \\ UCSD \\ nicola@cs.ucsd.edu \\ Jerome Simeon \\ IBM T.J. Watson Research \\ simeon@us.ibm.com
}

\begin{abstract}
Many modern applications, from Grid computing to RSS handling, need to support data processing in a distributed environment. Currently, most such applications are implemented using a general purpose programming language, which can be expensive to maintain, hard to configure and modify, and require hand optimization of the distributed data processing operations. We present Distributed XQuery (DXQ), a simple, yet powerful, extension of XQuery to support distributed applications. This extension includes the ability to deploy networks of XQuery servers, to remotely invoke XQuery programs on those servers, and to ship code between servers. Our demonstration presents two applications implemented in DXQ: the resolution algorithm of DNS, the Domain Name System, and the Narada overlay-network protocol. We show that our system can flexibly accommodate different patterns of distributed computation and present some simple but essential distributed optimizations.
\end{abstract}

Categories and Subject Descriptors: H.2 [Database Management]: Languages; D.1.3 [Software]: Programming Techniques-Concurrent Programming

General Terms: Languages,Reliability,Performance

Keywords: Distribution,XML,XQuery,Protocols

\section{INTRODUCTION}

Distributed data management represents the next frontier for database technology. Many modern applications, from Grid computing to RSS handling, require infrastructure that goes beyond the traditional client-server paradigm upon which traditional database management systems are built. They promise many benefits, like high performance by harnessing the power of under-utilized networked resources, reliability by leveraging the inherent redundancy of resources in a network, and concurrency by distributing computation simultaneously over multiple resources. Such promises cannot be fulfilled, however, without reliable infrastructure for configuring and managing heterogeneous components in a distributed system. The goal of the DXQ project is to support the development of reliable, extensible, and efficient distributed, data-intensive, applications.

We present Distributed XQuery (DXQ), a simple, yet powerful, extension of XQuery to support distributed applications. This extension includes the ability to deploy

Copyright is held by the author/owner(s).

SIGMOD'07, June 11-14, 2007, Beijing, China.

ACM 978-1-59593-686-8/07/0006. networks of XQuery servers, the ability to call XQuery programs on those servers, and to ship code between servers. Our demonstration presents two applications implemented in DXQ: the resolution algorithm of DNS, the Domain Name System, and the Narada overlay-network protocol [6]. DNS and Narada are representative of more complex distributed applications and overlay networks, e.g., for resource management $[4,11]$, or multicast [6]. Those protocols are usually implemented in a general purpose programming language, which make them expensive to maintain and hard to optimize.

With more of data processed by distributed systems being in XML, we believe DXQ provides a natural framework for implementing such systems. The DXQ code for DNS resolution is less than 100 lines of code, while a typical $\mathrm{C}$ implementation is $5000+$ lines of code. DXQ thus enables rapid development and makes programs easier to modify and maintain. DXQ also implements distributed query optimization with minimal developer effort. Finally, DXQ enables dynamic optimization not only of queries but also of the location at which queries will execute.

\section{Demonstration}

Our demonstration introduces DXQ's distributed features, shows how they can be used to support highly distributed applications in a simple and maintainable way, and illustrates some simple but essential distributed optimizations. We demonstrate both the DNS and Narada applications, in two parts. In the first part, we will show a movie of each application running on a network of ten DXQ servers recorded in our lab at AT\&T. The movie visualizes inter-server message traffic and application-specific changes of state. The movie is generated from a trace of inter-server messages produced by DXQ servers. In the second part, we give an interactive demonstration and show a live movie of each application running on a virtual network simulated by multiple processes running on a single machine. Distributed applications are hard to understand and debug, and we find that the visualization is critical to explaining a DXQ application's semantics and optimized behavior.

Here, we describe several scenarios of the DNS application that will be demonstrated. A demonstration of the Narada application can be found at: http://db.ucsd.edu/dxq/.

Iterative DNS Resolution. Resolution is the process of looking up an address for a hostname by consulting various nameservers; in DXQ, it is easily accomplished with a simple iterative query.

Optimized Iterative Resolution. We improve performance 
through distributed optimization, notably, combining multiple queries and pushing joins from client to server.

Recursive Resolution. We show how to simply implement a different distribution pattern in which the servers recursively call each other, off-loading work from the client.

Caching Resolution. This scenario demonstrates how XML updates [3] can implement caching for DNS servers.

Multicast. This is the most advanced scenario, where we demonstrate how DXQ can distribute a query to execute in parallel on many nodes in the network. This requires asynchrony and is essential to scale such applications to a large number of nodes.

DXQ is implemented on top of the Galax XQuery 1.0 processor, and it is publicly available as part of Galax's latest release. Due to space limitations, this proposal only briefly describes the code and features of the DNS application. A complete description of the various scenarios that will be demonstrated, the corresponding DXQ code, and movies that illustrate the application running, can be found at: http://db.ucsd.edu/dxq/.

Related Work. Most of the work in the area of distributed databases [10] focuses on distributed query processing in a controlled environment with a limited number of nodes. Work in the area of peer to peer networks (e.g., [13]) focuses on algorithms for efficient distribution of queries and is complementary to our work, which focuses on the query distribution infrastructure. DXQ's remote execution operator was inspired by Jim's previous work on using distributed databases for security policies [5, 7]. XQueryD [12] extended XQuery with exception handling and an operator identical to our synchronous exec; DXQ currently lacks exception handling, but includes asynchronous exec and XML updates. Sophia [14] is an extension of Prolog with a feature like our exec. In comparison to DXQ, Sophia includes continuous update, but lacks asynchronous execution and XML support. NDlog [8] and SeNDlog [1] are logic languages, descended from Datalog, that support declarative specification of distributed network and security protocols. Like DXQ, their goal is to make development of distributed network applications more reliable, extensible, and efficient. Despite the wealth of optimization techniques for rule-based languages, their applicability is limited. Implementation of many features necessary in distributed protocols is convoluted: updating counters and protocol state, and APIs to host languages are cumbersome, and the absence of modularity makes programs exceeding fifty or more rules hard to understand and modify. Our goal is for DXQ to hit the "sweet spot" between general-purpose programming languages and telegraphic rule-based languages.

\section{DXQ OVERVIEW}

The DXQ language includes all of XQuery 1.0 with updates $[2,3]$ and extends it in two ways. To the query pro$\log$, it adds an import-interface declaration, which permits a client query to remotely call functions in a module exported by a DXQ server. To the expression language, it adds a remote-execute expression in the style of [12], which redirects evaluation of an expression to a DXQ server. The following grammar gives the syntax for these extensions, which are illustrated below.

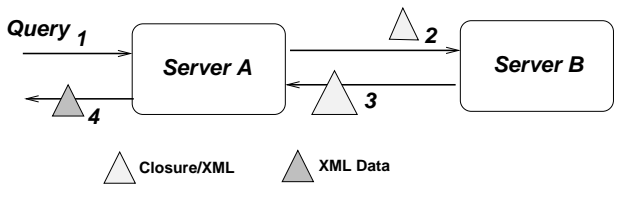

Figure 1: DXQ Servers

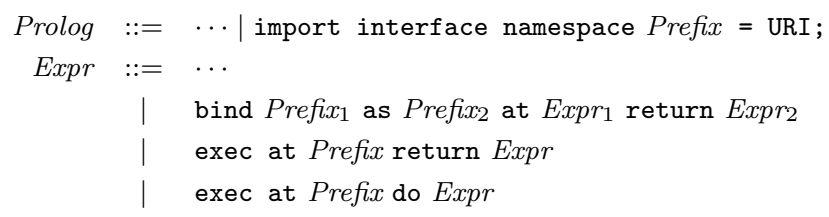

The bind expression associates an interface with an implementation on a given server (specified by the expression after the at clause). The implementation is identified by a new namespace prefix (Prefix $x_{1}$ ), which is in scope for the expression after the return clause $\left(\operatorname{Expr}_{2}\right)$. The two exec at expressions ship a query (Expr) to a given location synchronously (with the return clause) or asynchronously (with the do clause). A synchronous exec evaluates to the result of the remote execution, while an asynchronous exec returns the empty sequence immediately.

\subsection{DXQ Architecture}

Much like a Web server exports an interface of permissible messages, a $D X Q$ server exports a module of XQuery functions that may be called remotely from a client application or from another DXQ server. Unlike a Web server, a DXQ server can evaluate any XQuery expression submitted by a requester, which may include calls to the server's exported functions. The ability to move arbitrary queries between DXQ servers enables distributed query optimization, which can reduce the number of messages between servers and the amount of data moved between servers that are necessary to evaluate a query. In response to a request, a DXQ server may evaluate an expression and return an extensional XML value. In addition, a DXQ server may return an intensional XQuery expression that the requester may evaluate to compute the result itself. This mechanism enables a flexible range of resource-management policies to be applied in a distributed system.

Figure 2.1 shows the basic interaction of two DXQ servers, which can be generalized to any number. Server A accepts an XQuery expression from a user application (Step 1), compiles that query given the server's module context, evaluates the resulting query, and returns an XML value (Step 4). To compute the result may require that some fragment of the compiled query be evaluated by Server B, in which case Server A constructs a closure that encapsulates the query fragment and ships the closure to Server B (Step 2). Similarly, Server B compiles the closure in its own context and returns a result to Server A (Step 3).

\section{IMPLEMENTING DNS WITH DXQ}

Due to space limitations, we only illustrate parts of the first and fourth scenarios listed at the beginning of this proposal. DNS [9] provides the basic infrastructure for name resolution on the Internet, mapping a hostname to its IP address. DNS name servers are organized as a distributed tree. 
Each name server exports a database of resource records that contains the IP address of a hostname or a delegation indicating that another nameserver is responsible for the IP address of a hostname. In DXQ, every peer server first exports the following XQuery module interface, which contains one function that returns all of a server's resource records:

module namespace Server = "DNSServer";

declare function Server:RR() external;

The Server: RR function returns local DNS resource records. For example, the module below is the start of authority for research.att.com, and includes the hostname and IP address for the "root" nameserver:

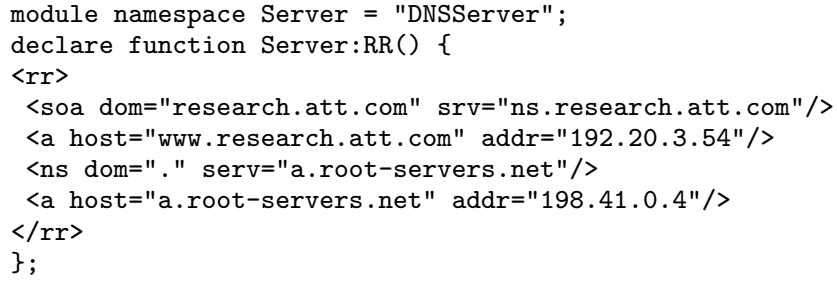

With a network of servers in place, organizations can run a DNS resolver which can contact known servers in order to resolve names requested by users. Using DXQ, the DNS resolver algorithm can be implemented by the following recursive function:

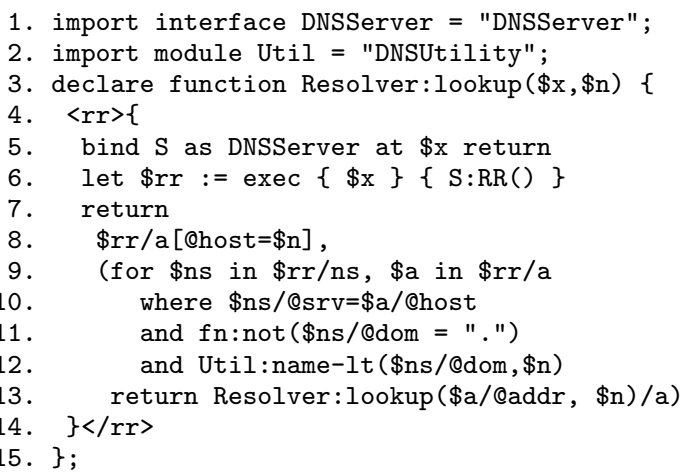

The query contains one exec expression (Line 6), and the server $X$ returns all its resource records to the requester. The result includes all address records for host $H$ defined by server $X$ (Line 8 ), and the address records defined by nameservers known to $X$ (Lines 9-13), which requires calling lookup recursively (Line 13). This particular implementation is iterative, i.e., one resolving server contacts all the delegating servers until one of them returns an IP address for the name, but the servers do not contact each other. A more complex scenario is for the servers to contact each other through recursive distributed calls and propagate the result back to the resolving server.

\subsection{DNS with caching}

Updates in XQuery [3] can be used to support advanced DNS features, such as caching. For example, to avoid redundant computation, the following version of lookup maintains a cache of hostname, address pairs, indexed by server:

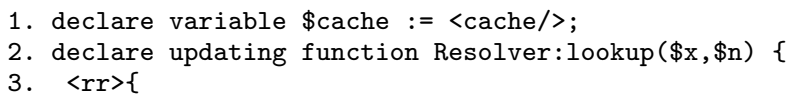

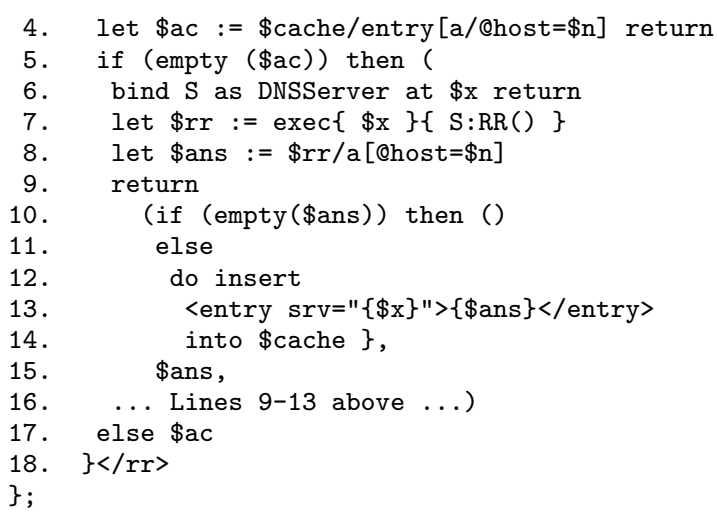

Note that Lines $5-14$ first check whether the name requested is in the cache and if it is not, executes a lookup and updates the cache. XQuery with updates can also support more advanced caching policies. At the conference, we plan to demonstrate other DNS features as well, such as expiration of cache entries, batch lookup of hostnames, and conditional protocols that depend on the requester's IP address. Likewise, we'll present in detail the multicast scenario. All these features are implemented concisely and transparently in DXQ.

\section{REFERENCES}

[1] M. Abadi and B. T. Loo. Towards a declarative language and system for secure networking. In NetDB '07: Proceedings of the 3rd International Workshop on Networking meets Databases, 2007.

[2] S. Boag, D. Chamberlin, M. F. Fernández, D. Florescu, J. Robie, and J. Simeon. XQuery 1.0: An XML query language. Technical report, World Wide Web Consortium, Nov. 2006. http://www.w3.org/TR/xquery.

[3] D. Chamberlin, D. Florescu, and J. Robie. XQuery update facility. Technical report, World Wide Web Consortium, July 2006. http://www.w3.org/TR/xqupdate/.

[4] I. Foster, H. Kishimoto, A. Savva, D. Berry, A. Grimshaw, B. Horn, F. Maciel, F. Siebenlist, R. Subramaniam, J. Treadwell, and J. V. Reich. The open grid services architecture, version 1.5. Technical Report GFD.80, Global Grid Forum, 2006

http://www.ggf.org/documents/GFD.80.pdf.

[5] C. A. Gunter and T. Jim. Policy-directed certificate retrieval. Software Practice and Experience, 30(15), 2000.

[6] Y. hua Chu, S. Rao, S. Seshan, and H. Zhang. A case for end system multicast. IEEE Journal on Selected Areas in Communication (JSAC), 20(8), 2002.

[7] T. Jim and D. Suciu. Dynamically distributed query evaluation. In PODS, 2001.

[8] B. T. Loo, T. Condie, M. Garofalakis, D. E. Gay, J. M. Hellerstein, P. Maniatis, R. Ramakrishnan, T. Roscoe, and I. Stoica. Declarative networking: language, execution and optimization. In SIGMOD '06: Proceedings of the 2006 ACM SIGMOD international conference on Management of data, pages 97-108, New York, NY, USA, 2006. ACM Press.

[9] P. Mockapetris. Domain names - concepts and facilities. http://www.ietf.org/rfc/rfc1034.txt, 1987.

[10] M. T. Ozsu and P. Valduriez. Principles of distributed database systems. Prentice-Hall, 1991.

[11] L. Peterson and J. Wroclawski. Overview of the GENI architecture. Technical Report Design Document 06-11, Global Environment for Network Innovations, 2006. http://www.geni.net/GDD/GDD-06-11.pdf.

[12] C. Re, J. Brinkley, K. Hinshaw, and D. Suciu. Distributed XQuery. In Workshop on Information Integration on the $W e b$, pages $116-121,2004$.

[13] I. Tatarinov et al. The Piazza peer data management project. SIGMOD Records, 32(3):47-52, 2003.

[14] M. Wawrzoniak, L. Peterson, and T. Roscoe. Sophia: an information plane for networked systems. SIGCOMM Comput. Commun. Rev., 34(1):15-20, 2004. 